\title{
Complete Sequence and Analysis of the Mitochondrial Genome of Hemiselmis andersenii CCMP644 (Cryptophyceae) Eunsoo Kim ${ }^{1}$, Christopher E Lane ${ }^{1}$, Bruce A Curtis ${ }^{2}$, Catherine Kozera ${ }^{2}$, Sharen Bowman ${ }^{2,3}$ and John M Archibald*1
}

Address: ${ }^{1}$ Canadian Institute for Advanced Research, Integrated Microbial Biodiversity Program, Department of Biochemistry and Molecular Biology, Dalhousie University, Halifax, Nova Scotia, Canada, ${ }^{2}$ The Atlantic Genome Centre, Halifax, Nova Scotia, Canada and ${ }^{3}$ Department of Process Engineering and Applied Science, Dalhousie University, Halifax, Nova Scotia, Canada

Email: Eunsoo Kim - eunsookim@dal.ca; Christopher E Lane - C.Lane@dal.ca; Bruce A Curtis - Bbcurtis@genomeatlantic.ca; Catherine Kozera - Catherine.Kozera@nrc-cnrc.gc.ca; Sharen Bowman - Sharen.Bowman@nrc-cnrc.gc.ca; John M Archibald* - jmarchib@dal.ca

* Corresponding author

Published: 12 May 2008

BMC Genomics 2008, 9:215

doi: $10.1|86 /| 47|-2| 64-9-215$

Received: 19 February 2008

Accepted: 12 May 2008

This article is available from: http://www.biomedcentral.com/I47I-2/64/9/2I5

(C) 2008 Kim et al; licensee BioMed Central Ltd.

This is an Open Access article distributed under the terms of the Creative Commons Attribution License (http://creativecommons.org/licenses/by/2.0), which permits unrestricted use, distribution, and reproduction in any medium, provided the original work is properly cited.

\begin{abstract}
Background: Cryptophytes are an enigmatic group of unicellular eukaryotes with plastids derived by secondary (i.e., eukaryote-eukaryote) endosymbiosis. Cryptophytes are unusual in that they possess four genomes-a host cell-derived nuclear and mitochondrial genome and an endosymbiont-derived plastid and 'nucleomorph' genome. The evolutionary origins of the host and endosymbiont components of cryptophyte algae are at present poorly understood. Thus far, a single complete mitochondrial genome sequence has been determined for the cryptophyte Rhodomonas salina. Here, the second complete mitochondrial genome of the cryptophyte alga Hemiselmis andersenii CCMP644 is presented.

Results: The $H$. andersenii mtDNA is 60,553 bp in size and encodes 30 structural RNAs and 36 protein-coding genes, all located on the same strand. A prominent feature of the genome is the presence of a $\sim 20 \mathrm{Kbp}$ long intergenic region comprised of numerous tandem and dispersed repeat units of between 22-336 bp. Adjacent to these repeats are 27 copies of palindromic sequences predicted to form stable DNA stem-loop structures. One such stem-loop is located near a GCrich and GC-poor region and may have a regulatory function in replication or transcription. The $H$. andersenii mtDNA shares a number of features in common with the genome of the cryptophyte Rhodomonas salina, including general architecture, gene content, and the presence of a large repeat region. However, the $H$. andersenii mtDNA is devoid of inverted repeats and introns, which are present in $R$. salina. Comparative analyses of the suite of tRNAs encoded in the two genomes reveal that the $H$. andersenii mtDNA has lost or converted its original trnK(uuu) gene and possesses a trnSderived 'trnK(uuu)', which appears unable to produce a functional tRNA. Mitochondrial protein coding gene phylogenies strongly support a variety of previously established eukaryotic groups, but fail to resolve the relationships among higher-order eukaryotic lineages.
\end{abstract}

Conclusion: Comparison of the $H$. andersenii and $R$. salina mitochondrial genomes reveals a number of cryptophyte-specific genomic features, most notably the presence of a large repeat-rich intergenic region. However, unlike $R$. salina, the $H$. andersenii mtDNA does not possess introns and lacks a Lys-tRNA, which is presumably imported from the cytosol. 


\section{Background}

The mitochondrion is a double-membrane enclosed organelle found in the vast majority of extant eukaryotes. Mitochondria are best known for their essential role in energy generation, but they are also the site of additional important cellular processes such as iron-sulfur ( $\mathrm{Fe}-\mathrm{S}$ ) cluster assembly and the beta-oxidation of fatty acids [1]. Some degenerate forms of mitochondria, such as the mitosome of the diplomonad parasite Giardia lamblia, have secondarily lost energy generating pathways and seem to retain only the $\mathrm{Fe}$-S cluster maturation function [2]. All mitochondria are believed to share a single origin from an $\alpha$-proteobacterial-like prokaryote [1], but a wide diversity of mitochondrial genome architectures have evolved subsequent to the diversification of modern-day eukaryotes $[1,3,4]$. For example, whereas "derived" animals possess monomeric circular mitochondrial genomes, an observation which led to the initial assumption that mtDNAs are primarily circular [5], many other mitochondrial genomes, such as that of the ciliate Tetrahymena pyriformis [6], the green alga Chlamydomonas reinhardtii [7] and the cnidarian metazoan Aurelia aurita (moon jelly) [8] are linear [9]. In addition, while some fungi and many plants have circular-mapping mtDNAs, their mitochondria actually contain predominantly linear mtDNA molecules with combinations of monomers and concatemers, with only a minor fraction of the molecules present in a circular form [10]. A more extreme example is the mtDNA of kinetoplastids, which consists of one maxiand many different mini-circles that are interconnected to form an extensive network [11]. Mitochondrial gene content is also highly variable; the mtDNA of the jakobid flagellate Reclinomonas americana encodes 97 genes, the largest set of mitochondrial genes currently known [12], whereas the mtDNA of the malaria parasite Plasmodium falciparum contains just 3 protein coding genes and 2 highly fragmented small and large subunit ribosomal RNA (rRNA) genes [13]. The most highly derived forms of mitochondria, such as the hydrogenosome of Trichomonas vaginalis [14] and the Giardia lamblia mitosome [2], have lost their genomes entirely [15].

Mitochondria are also known as sites of unusual molecular biology and biochemistry. Marande and Burger [3] recently showed that the mtDNA genes of the euglenid Diplonema papillatum are fragmented into as many as nine modules, each residing on a distinct 6 or 7 Kbp chromosome. The mechanism by which these fragmented gene pieces are linked together to form contiguous transcripts is unknown. Extensive mRNA editing is another example of the bizarre molecular biology of mitochondria. Kinetoplastid mitochondrial mRNAs are subject to insertions and deletions of uridylate residues, sometimes $>100$ such insertions/deletions per transcript [16]. Mitochondrial mRNA editing is also widespread in land plants [17] and dinoflagellates [18]. For example, $2 \%$ of the cox 1 and $c o b$ gene sequences in three dinoflagellate species investigated by Lin et al. [19] were edited at the mRNA level.

We are studying the genomic diversity and evolution of cryptophytes, a ubiquitous and ecologically significant group of single-celled eukaryotes found in freshwater and marine environments. Most cryptophytes, except for members of the genus Goniomonas, harbor plastids of secondary endosymbiotic origin [20]. A variety of shared morphological features, such as the presence of ejectisomes, flat mitochondrial cristae, and an anterior depression, support the monophyly of cryptophytes, as do molecular phylogenetic data [21]. One unique feature of cryptophyte plastids that distinguishes them from other plastids of red algal origin is the retention of the remnant nucleus of the red algal endosymbiont, referred to as the nucleomorph [22,23]. Consequently, most cryptophytes harbor four distinct genomes-nuclear, nucleomorph, mitochondrial, and plastid genomes-contained in separate compartments. Cryptophytes are thus an interesting model system with which to study endosymbiotic gene transfer, genome evolution, and protein targeting.

In this study, we report the complete mitochondrial genome sequence of the newly described cryptophyte species Hemiselmis andersenii CCMP644, and compare it to the only other cryptophyte mitochondrial genome described thus far, that of Rhodomonas salina [24]. In addition, individual and concatenated mitochondrial protein coding gene sequences were analyzed to infer the phylogenetic relationships of cryptophytes to other eukaryotes.

\section{Methods DNA preparation, sequencing, and genome assembly}

Hemiselmis andersenii mtDNA was isolated and sequenced to $\sim 10 \times$ coverage as described in Lane et al. [25]. About 1,200 end sequences were screened for quality and vector contamination with Pregap4 and automatically assembled using gap4 version 4.10 in the Staden package [26]. Complete automated assembly of a large intergenic space between trnS and $\operatorname{cox} 2$ was unsuccessful due to the highly repetitive nature of this region. In an attempt to manually resolve this area, short ( $30 \mathrm{bp}$ ) unique sequences within the trnS and cox 2 genes were used to probe the sequence database for reads that extended from these two loci into the repeat region. These sequences were extracted and manually aligned using MacClade version 4.08 [27]. Sequences at the ends of the new constructs were then selected and the process was repeated. However, due to the presence of multiple identical copies of a >500 bp repeat, the assembly of a single unambiguous contig was not possible. When all available sequence reads were considered, three robust contigs were produced, each ending with similar repetitive sequences consisting of a $\sim 340 \mathrm{bp}$ 
repeat unit. These three contigs were joined to circularize the map. The complete $H$. andersenii mtDNA has been submitted to GenBank under the following accession number: EU651892.

DNA secondary structure within the repeat region was predicted using mfold version 3.2 [28] at a folding temperature of $37^{\circ} \mathrm{C}$ and the ionic conditions of $1.0 \mathrm{M}\left[\mathrm{Na}^{+}\right]$ and $0.0 \mathrm{M}\left[\mathrm{Mg}^{++}\right]$.

\section{Genome size/structure determination}

We used pulsed-field gel electrophoresis (PFGE) to obtain an independent size estimate of the $H$. andersenii mitochondrial genome. Hemiselmis andersenii total DNA plugs were prepared as described in Lane et al. [29] and digested overnight with the restriction enzymes PstI or BglII (Fermentas, Hanover, MD, USA). Based on the genome sequence, these enzymes were predicted to cut the mtDNA only once or twice. Both untreated and enzyme-digested $H$. andersenii DNA plugs were run on a $1 \%$ agarose gel $(1 \times$ $\mathrm{TBE}$ ) in $0.5 \times \mathrm{TBE}$ buffer at $14.0^{\circ} \mathrm{C}$ for $18 \mathrm{~h}$ at a voltage of $6.0 \mathrm{~V} / \mathrm{cm}$ with a switch time between 1-25 s using a CHEF-DR III Pulsed-Field Gel Electrophoresis System (Bio-Rad Laboratories, Hercules, CA, USA). DNA on the pulsed-field gel was transferred to a nylon membrane. Southern hybridization using a 700 bp coxI probe as in Lane and Archibald [30] revealed that undigested mitochondrial DNA molecules were trapped in the wells or found in the 'compression zone'. The PstI or BglII endonuclease treated DNA plugs revealed mitochondrial molecules in a discrete band below the 'compression zone'. The corresponding bands could not be visualized on the ethidium-bromide stained pulsed-field gel image because of nuclear and nucleomorph DNA smears in the background. In order to visualize mtDNA on the pulsed-field gel, an initial PFGE run was used to remove the linear nuclear and nucleomorph chromosomes from the PFGE plugs. These plugs, which still contained organellar DNA, were subsequently removed from the gel and digested with the restriction enzymes PstI and BglII. Digested plugs were then inserted into a fresh gel and electrophoresed under the conditions described above. The $5 \mathrm{Kbp}$ and Lambda CHEF DNA Size Standard (Bio-Rad Laboratories, Hercules, CA, USA) were used to estimate the size of the enzymatically linearized $H$. andersenii mtDNA.

\section{Genome annotation and GC content/skew analyses}

Annotation of the $H$. andersenii mtDNA and the GC content and skew analyses were performed in Artemis version 8 [31]. Gene identification was carried out using BLASTX and BLASTN. Small and large ribosomal rRNA subunit genes were identified by comparison to rRNA gene sequences in the mitochondrial genome of Rhodomonas salina. Transfer RNAs were identified using tRNAscan-SE version 1.21 [32].

\section{Genome rearrangements between the two cryptophyte mtDNA}

The extent to which the $H$. andersenii and $R$. salina mitochondrial genomes are rearranged to each other was estimated using GRIMM [33]. Each genome was designated as a sequence of 63 units, which include a repeat region and 62 genes common between the two cryptophyte mtDNAs.

\section{RT-PCR of 'trnK(uuu)'}

tRNAscan-SE version 1.21 [32] identified a putative intron of $\sim 20 \mathrm{bp}$ in the anticodon loop of the $H$. andersenii trnK(uuu) gene. To determine whether this prediction was correct, we performed RT-PCR using Lysine-tRNA-specific primer pairs and $H$. andersenii total RNA provided by $H$. Khan. To eliminate DNA contamination, $1 \mu \mathrm{l}$ of total RNA was incubated for $30 \mathrm{~min}$ with RQ1 RNase-Free Dnase (Promega, Madison, WI, USA). RT-PCR was performed using the QIAGEN one-step RT-PCR kit (QIAGEN, Valencia, CA, USA) and with control reactions in which the reverse-transcription process was skipped. The following two pairs of primers were used: 1 ) The forward primer 5'GAAGGTTGCTCGAATGGAA-3' with the reverse primer 5'-GAAGGTATAGGAATTGAACCTATTC-3' 2) and the forward primer 5'-GCCCAGAAGGTTGCTC-3' with the reverse primer 5'-AAGAAGGTATAGGAATTGAACCTAT-3'. RT-PCR was performed with the reverse transcription step for $30 \mathrm{~min}$ at $50^{\circ} \mathrm{C}$ and the subsequent inactivation of reverse transcriptase and activation of HotStart Taq DNA polymerase for $15 \mathrm{~min}$ at $95^{\circ} \mathrm{C}$, followed by 35 cycles at $94^{\circ} \mathrm{C}$ for $1 \mathrm{~min}, 47^{\circ} \mathrm{C}$ for $1 \mathrm{~min}$, and $72^{\circ} \mathrm{C}$ for $1 \mathrm{~min}$, and a final extension at $72^{\circ} \mathrm{C}$ for $10 \mathrm{~min}$. The amplified PCR fragments were cloned into pCR4-TOPO vector in the TOPO TA cloning kit for sequencing (Invitrogen, Carlsbad, CA, USA). Between 5 and 10 bacterial colonies from each reaction were selected for sequencing on a Beckman Coulter CEQ8000 (Beckman Coulter Inc., Fullerton, California, USA).

\section{Molecular phylogenetic analysis}

From the 36 protein-coding genes found in the $H$. andersenii mtDNA, 25 were selected for phylogenetic analyses. Eleven genes (atp8, nad8, rps2, rps3, rps4, rps7, rps8, rps13, rpl5, rpl6, tatC) were excluded because their sequences were poorly conserved and/or were only present in a few taxonomic groups. $H$. andersenii protein sequences were aligned with their homologs from other mitochondrial genomes available from GenBank. Amino acid sequences were aligned using MacClade version 4.08 [27] and ambiguously aligned sites were manually removed. In addition to individual protein analyses, a concatenated protein data set containing 25 proteins was analyzed. To include the maximum number of gene sequences, we combined 25 protein-coding gene sequences encoded in 18 mitochondrial genomes across diverse eukaryotic taxa. 
As most mitochondrial genomes do not possess all 25 protein-coding genes selected for analysis, as many as 12 protein gene sequences were missing per taxon. A maximum likelihood tree was produced using RAxML-VI-HPC version 2.2.3 [34] with the PROTOMIXJTT model of sequence evolution and the automatic tree rearrangement setting, and from 100 distinct randomized maximum parsimony starting trees. Bootstrap analysis was based on 100 re-samplings.

\section{Results and Discussion General features of Hemiselmis andersenii mtDNA}

The mitochondrial DNA of the cryptophyte Hemiselmis andersenii CCMP644 was sequenced, assembled and manually edited to produce a circular-mapping genome $60,553 \mathrm{bp}$ in size (Figure 1). Genome assembly was complicated by the presence of a highly repetitive non-coding region of $20 \mathrm{Kbp}$ (see below); genome size was thus verified using pulsed-field gel electrophoresis (PFGE). Several observations suggest that the $H$. andersenii mtDNA exists primarily in a linear-branched form comprised of multiple genome units. In PFGE, the H. andersenii mtDNA remains in the well or migrates within the 'compression zone' (i.e., the unresolved portion of DNA near the top of the gel), which contains primarily linear nuclear and nucleomorph chromosomes larger than 150 Kbp (data not shown). The lack of mtDNA below the 'compression zone' suggests that the $H$. andersenii mtDNA is not composed of linear monomers or dimers. Furthermore, when the $H$. andersenii mtDNA is partially digested with PstI, an enzyme predicted to cut the genome only once, it produces a discrete band of $\sim 60 \mathrm{Kbp}$ in size (data not shown) but not a band $\sim 120 \mathrm{Kbp}$ in size, which would correspond to a dimeric linear form of the genome. This result indicates that the H. andersenii mtDNA is not composed of circular concatemers or linear head-to-tail concatemers consisting of three or more genomic units. Therefore, we suggest that the $H$. andersenii mtDNA exists primarily as a branched linear molecule although monomeric circles may also exist. Further studies using transmission electron microscopy or the 'moving picture' technique [10] will be necessary to confirm this hypothesis.

The $H$. andersenii mitochondrial genome is comprised of a gene-rich region $\sim 40 \mathrm{Kbp}$ in size and a large $(19,675 \mathrm{bp})$ intergenic region between trnS and cox 2 with complex repeats (Figures 1 and 2). The intergenic region accounts for $32.5 \%$ of the entire genome and $83.5 \%$ of the total amount of non-coding DNA (23,549 bp). The overall GC content of the genome is $28.72 \%$, slightly higher than that of the nucleomorph genome of this organism [25]. Interestingly, a $40 \mathrm{bp}$ region near the start of the coding portion of the genome is very GC-rich $(78.38 \%)$ and is followed by a $100 \%$ AT-containing region 190 bp in size (Figure 3). This unusual stretch of sequence is about $70 \mathrm{bp}$ from a palindromic sequence that is predicted to form a Type II stem-loop (Figures 2 and 3; see discussion below), and may be involved in regulating replication or transcription.

The $H$. andersenii mitochondrial genome encodes 66 genes with predicted functions and 8 hypothetical protein-coding genes, a total somewhat higher than the average for eukaryotes (40-50 genes) [1]. Ten genes-orf167, orf71, rps13, rps11, nad3, rps2, tatC, 'trnK (uuu)', rps12, and rps7-overlap by up to $51 \mathrm{bp}$, emphasizing the extreme compactness of the coding portion of the genome. The genome encodes small and large rRNA subunit genes and 28 tRNAs, one of which may be a pseudogene (see discussion below). Of the 36 identifiable protein-coding genes, 14 encode ribosomal proteins, 21 are involved in oxidative phosphorylation, and one gene encodes a membrane translocase protein (Table 1).

Comparison of the mtDNA gene order in H. andersenii to other genomes reveals the presence of five gene clusters shared among distantly related protists: two ribosomal protein clusters (rps12-rps7-rps19-rps3-rpl16-rpl14-rpl5$r p s 14$ and rps8-rpl6-rps13-rps11) and three NADH dehydrogenase clusters (nad4L-nad5; nad4-nad2; nad10-nad9). These gene clusters have been suggested to represent vestiges of bacterial operons $[12,24]$. Interestingly, all 74 genes in the $H$. andersenii mitochondrial genome are encoded on the same strand. While the evolution of such an arrangement seems improbable, absolute strand polarity has been observed in the mitochondrial genomes of diverse eukaryotes such as the amoeba Acanthamoeba castellanii (59 genes), the fungus Penicillium marneffei ( 47 genes), and the green alga Chlamydomonas eugametos (20 genes) [35-37]. In addition, strikingly similar mtDNA architectures-gene-dense regions, a single large repetitive intergenic region, and all genes encoded on one strandare seen in diverse protists such as the stramenopile Thraustochytrium aureum (The Organelle Genome Megasequencing Program; http://megasun.bch.umontreal.ca/ ogmp/) and the green alga Pedinomonas minor [38]. Understanding the biological significance of such convergence at the level of genome architecture will require comparative molecular and biochemical studies of mitochondria in these organisms.

\section{Comparison of the mitochondrial genomes of Hemiselmis andersenii and Rhodomonas salina}

$H$. andersenii is only the second cryptophyte, after $R$. salina [24], for which a mitochondrial genome has been completely sequenced and annotated. Comparative analyses of the two genomes revealed a number of similarities. Both genomes feature a compact gene arrangement and a single large repeat region (Figure 1) [24], although the size of the large intergenic region in H. andersenii ( $\sim 20 \mathrm{Kbp})$ is 


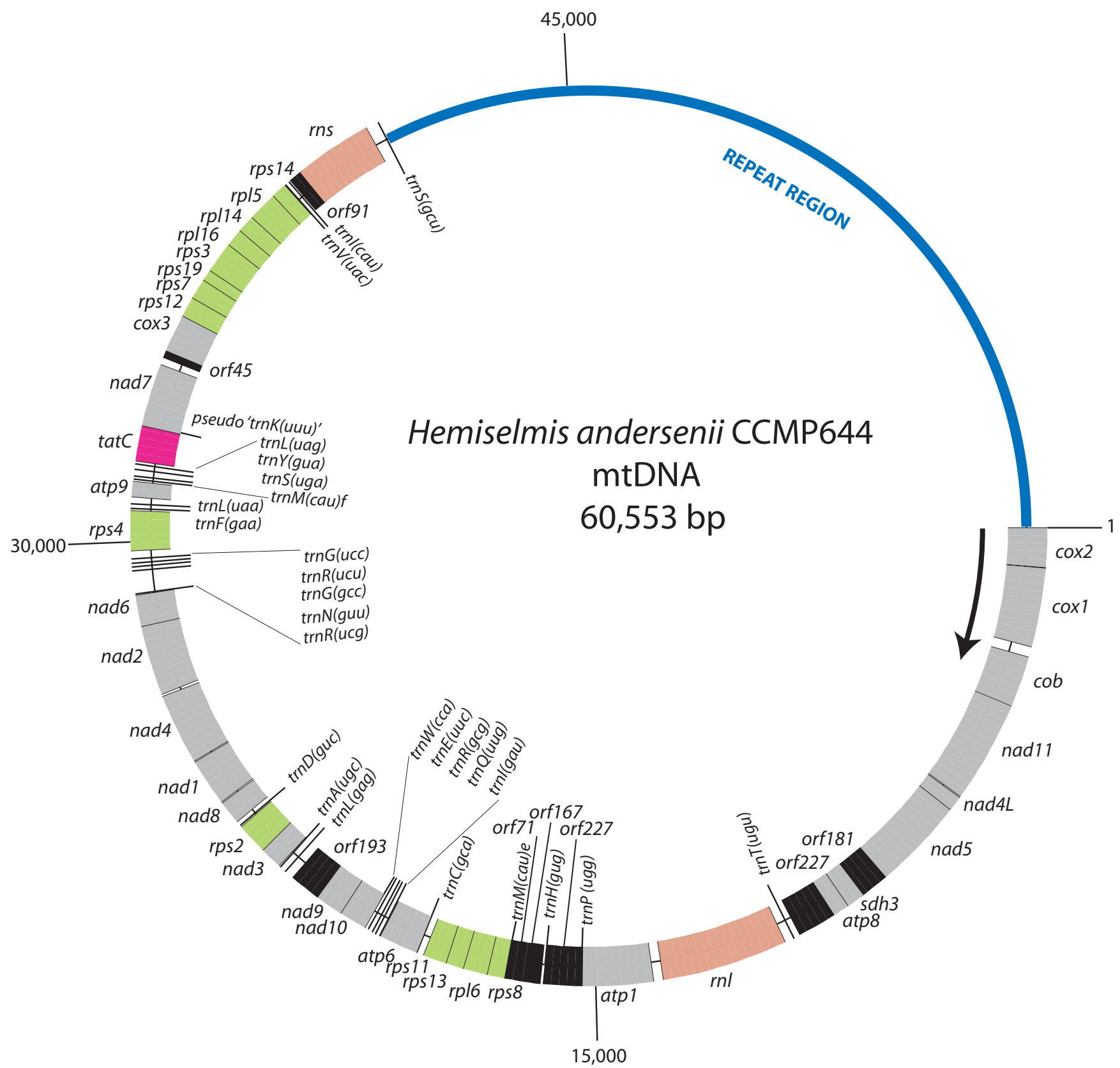

Figure I

Circular map of the mitochondrial genome of the cryptophyte Hemiselmis andersenii. All of the genes are transcribed in a clockwise direction. Note the dense gene arrangement and a single large intergenic region. Protein-coding genes and ribosomal RNA genes are labeled outside the circle, whereas transfer RNAs and open reading frames of unknown functions are labeled on the inside. 'TrnK(uuu)' may be a pseudogene (see main text for discussion). Genes are color-coded according to functional categories: green for ribosomal protein genes, gray for genes involved in oxidative phosphorylation, pink for the protein translocase protein gene tat $C$, salmon for ribosomal subunit genes, and black for open reading frames with unknown functions.

more than four times as large as that of $R$. salina ( $\sim 4.7$ $\mathrm{Kbp}$ ). All of the 36 predicted protein-coding genes in the $H$. andersenii mitochondrial genome are present in the $R$. salina mtDNA. Four $R$. salina mitochondrion-encoded genes-rps1, atp4, tatA, and sdh4-are not found in $H$. andersenii, although two open reading frames, orf 45 and orf91, in the $H$. andersenii mtDNA show marginal sequence similarity to the R. salina tatA and $s d h 4$ genes, 


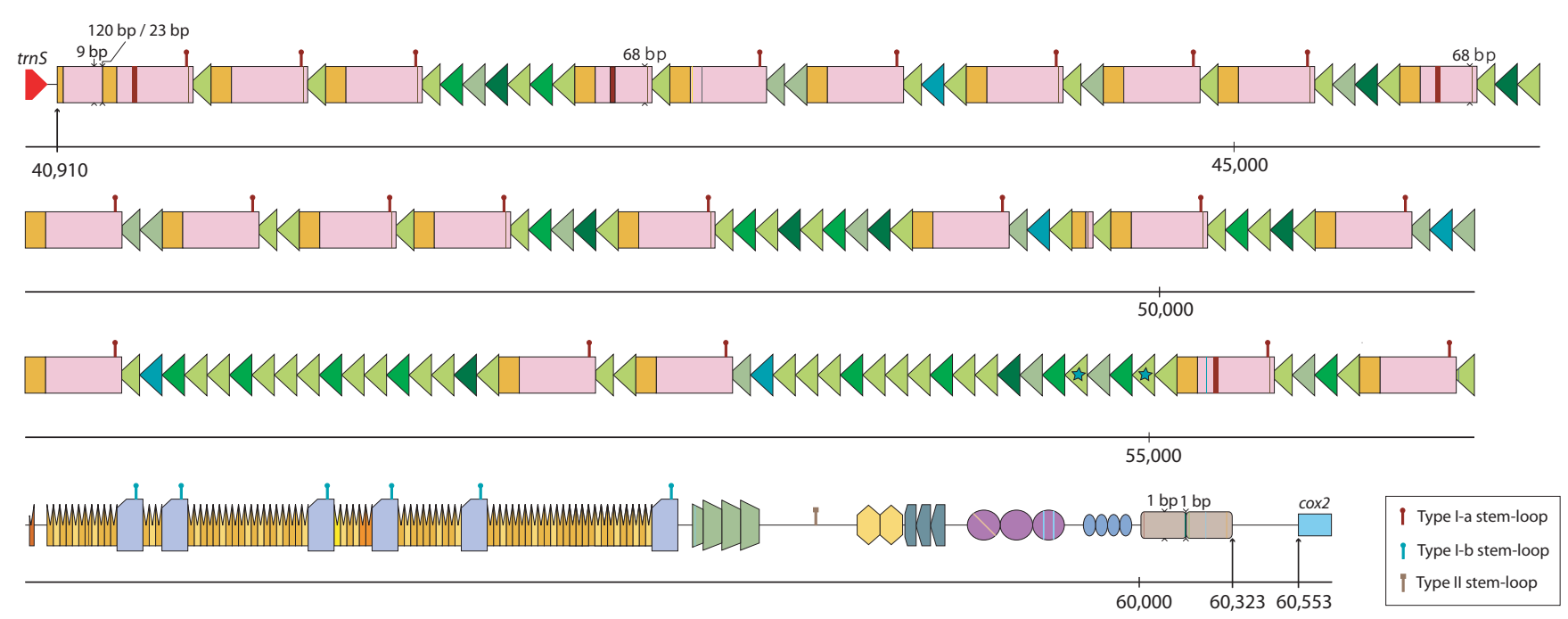

Figure 2

Schematic diagram of the large repeat region in the Hemiselmis andersenii mitochondrial genome. This region is $\sim 20 \mathrm{~Kb}$ in size and includes multiple repeat units arranged in tandem or dispersed among tandem repeats. Slight variations of each repeat unit are color-coded and/or marked with strips or a star symbol. Predicted nucleotide deletions within a repeat unit are highlighted with arrowheads: the size of the deletion is also provided. The positions of three kinds of DNA stem-loop forming sequences, Type I-a, I-b, and II, are labeled with "hairpin" symbols.

respectively. Additionally, while two group II introns are present in $R$. salina mtDNA, the $H$. andersenii mtDNA is devoid of introns (Table 2) [24].

With respect to conservation of gene order, $64.5 \%$ of the shared genes between the two cryptophyte mitochondrial genomes ( 40 out of 62 genes-36 protein-coding genes, 24 tRNA genes (see below), 2 rRNA genes) are present in thirteen syntenic blocks, each consisting of 2-7 genes. These include: 1) cox1-cob-nad11, 2) nad4L-nad5, 3) atp1$\operatorname{trnP}($ ugg), 4) rps8-rpl6-rps13-rps11, 5) $\operatorname{trnC}(g c a)-a t p 6,6)$ $\operatorname{trnI}($ gau $)-\operatorname{trn} Q(u u g)-\operatorname{trn} R(g c g)-\operatorname{trn} E(u u c)-\operatorname{trn} W(c c a)-$ nad10-nad9, 7) nad4-nad2, 8) trnR(ucu)-trnG(ucc), 9) $\operatorname{trnM}($ cau)f-trnS(uga), 10) $\operatorname{trnY}($ gua $)-\operatorname{trn} L($ uag), 11) tatC'trnK(uuu)' [H. andersenii] /trnS(gcu) [R. salina]-nad7, 12)cox3-rps12-rps7-rps19, and 13) rps3-rpl16-rpl14-rpl5rps14. As noted earlier, some of the conserved gene clusters, such as nad4L-nad5, are found in distantly related eukaryotes and appear to be vestiges of bacterial operons. Analysis using GRIMM [33] suggests that the observed difference in gene order between the two cryptophyte mitochondrial genomes can be explained by at least 31 instances of genome reversal events.

\section{Repeat structure of the $\mathbf{H}$. andersenii mitochondrial genome}

The R. salina mtDNA is characterized by a pair of $\sim 1.5 \mathrm{Kbp}$ inverted repeats that are joined by 112 bp of sequence [24]. In contrast, repeats in the H. andersenii mitochondrial genome are not inverted, but are instead dispersed or arranged in tandem throughout the large non-coding region, with individual repeat units ranging from 22 to $336 \mathrm{bp}$ and occurring up to 100 times (Figure 2). Given that $R$. salina and $H$. andersenii are distantly related to one another [29], the large repeat region presumably arose during or prior to the early diversification of cryptophytes. While there is no obvious sequence similarity between the two repeat regions, both contain multiple copies of palindromic sequences, which are predicted to form stable stem-loop DNA structures [24]. In H. andersenii, two types of stem-loop structures were identified-I and II-using the DNA MFOLD program [28]. The Type I structure has two slight variations, I-a and I-b, which occur 21 and 5 times, respectively (Figures 2 and 3 ). Type I-a and I-b structures have 22 and 20 base pairings in their stems, respectively, and occur adjacent to tandem repeats (Figures 2 and 3 ). One copy of the type II stem-loop structure is located within a 300 bp segment that is devoid of any discernable repeat units, but close to the high and low GC regions noted earlier (Figures 2 and 3). As was suggested for $R$. salina by Hauth et al. [24], tandem repeats and multiple stem-loop structures in $H$. andersenii mtDNA might be involved in the regulation of transcription and replication, a hypothesis that needs to be tested further.

Hauth et al. [24] demonstrated that the repeat region of the $R$. salina mtDNA roughly coincides with a change in the direction of 'cumulative GC skew' [calculated as (G$\mathrm{C}) /(\mathrm{G}+\mathrm{C})]$ and suggested that the repeat corresponds to the origin of replication. We investigated the GC skew in 
A

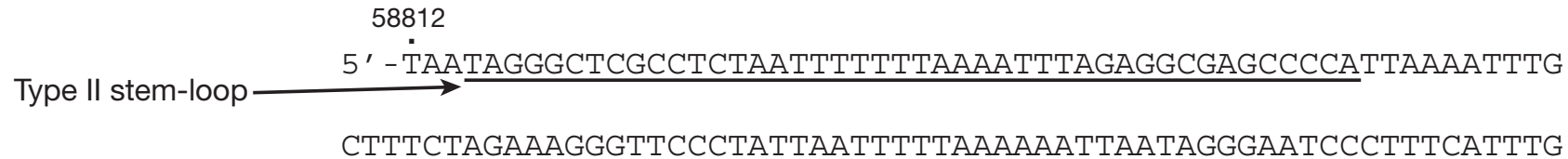

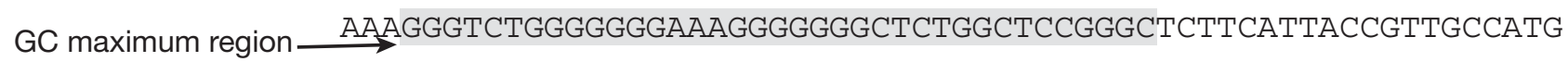

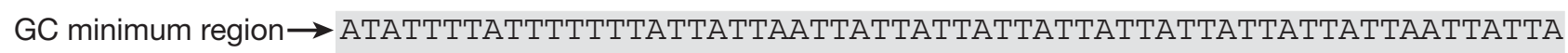

тТАТТАТТААТТАТТТТАТТАТТААТТАТТАТАТТАТТААТТАТТАТТАТТАТТАТТА

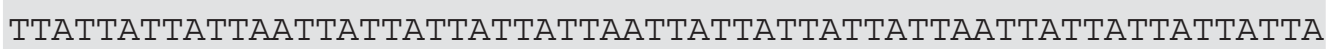

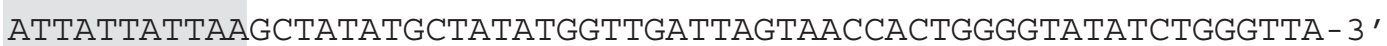

B

\begin{tabular}{|c|c|c|}
\hline${ }_{A} A_{T} A_{T}$ & & $\begin{array}{c}T \quad T \\
T \quad A \\
T-A\end{array}$ \\
\hline $\mathrm{A} \quad \mathrm{A}$ & $A \mathrm{~A} A$ & $\mathrm{~T}-\mathrm{A}$ \\
\hline $\mathrm{T}$ & $\mathrm{A}^{\mathrm{A}} \mathrm{T}$ & $\mathrm{T}-\mathrm{A}$ \\
\hline $\mathrm{C}_{\mathrm{T}-\mathrm{A}} \mathrm{T}$ & $\mathrm{T}$ & T T \\
\hline $\mathrm{G}-\mathrm{C}$ & $\mathrm{C}$ & $\begin{array}{l}A-T \\
A-T\end{array}$ \\
\hline $\mathrm{T}-\mathrm{A}$ & ${ }_{\mathrm{G}-\mathrm{C}}^{\mathrm{T}}$ & $\mathrm{T}-\mathrm{A}$ \\
\hline$C-G$ & $\mathrm{~T}-\mathrm{A}$ & $C-G$ \\
\hline $\mathrm{T}-\mathrm{A}$ & $C-G$ & $\mathrm{~T}-\mathrm{A}$ \\
\hline $\mathrm{T}-\mathrm{A}$ & $\mathrm{T}-\mathrm{A}$ & $\mathrm{C}-\mathrm{G}$ \\
\hline $\mathrm{T}-\mathrm{A}$ & $\mathrm{T}-\mathrm{A}$ & $C-G$ \\
\hline $\mathrm{T}-\mathrm{A}$ & $\mathrm{T}-\mathrm{A}$ & $\mathrm{G}-\mathrm{C}$ \\
\hline $\mathrm{A}-\mathrm{T}$ & $\mathrm{T}-\mathrm{A}$ & $\mathrm{C}-\mathrm{G}$ \\
\hline $\mathrm{G}-\mathrm{C}$ & $A-T$ & $\mathrm{~T}-\mathrm{A}$ \\
\hline $\mathrm{T}-\mathrm{A}$ & $\mathrm{G}-\mathrm{C}$ & $\mathrm{C}-\mathrm{G}$ \\
\hline $\mathrm{T}-\mathrm{A}$ & $\mathrm{T}-\mathrm{A}$ & $\mathrm{G}-\mathrm{C}$ \\
\hline $\mathrm{T}-\mathrm{A}$ & $\mathrm{T}-\mathrm{A}$ & $\mathrm{G}-\mathrm{C}$ \\
\hline $\mathrm{T}-\mathrm{A}$ & $\mathrm{T}-\mathrm{A}$ & $\mathrm{G}-\mathrm{C}$ \\
\hline $\mathrm{T}-\mathrm{A}$ & $\mathrm{T}-\mathrm{A}$ & A C \\
\hline $\mathrm{T}-\mathrm{A}$ & $\mathrm{T}-\mathrm{A}$ & $\mathrm{T}-\mathrm{A}$ \\
\hline $\mathrm{G}-\mathrm{C}$ & $\mathrm{T}-\mathrm{A}$ & $\mathrm{A}-\mathrm{T}$ \\
\hline $\mathrm{T}-\mathrm{A}$ & $\mathrm{G}-\mathrm{C}$ & $A-T$ \\
\hline $\mathrm{A}-\mathrm{T}$ & $\mathrm{T}-\mathrm{A}$ & $\mathrm{T}-\mathrm{A}$ \\
\hline $\mathrm{A}-\mathrm{T}$ & $A-T$ & $\mathrm{~T}-\mathrm{A}$ \\
\hline$A-T$ & $\mathrm{~A}-\mathrm{T}$ & $\mathrm{T}-\mathrm{A}$ \\
\hline $\mathrm{G}-\mathrm{C}$ & $\mathrm{A}-\mathrm{T}$ & $\mathrm{T}-\mathrm{A}$ \\
\hline Type I-a stem-loop & Type I-b stem-loop & Type II stem-loop \\
\hline
\end{tabular}

Figure 3

(A) Isolated region of the Hemiselmis andersenii mitochondrial genome located near the 3' end of the large intergenic space. This area includes a palindromic sequence that is predicted to form a Type Il stem-loop followed by high and low GC regions. A similar region is not found in the $R$. salina mtDNA. (B) Three predicted stem-loop structures found within the large intergenic space. Note that Type I-a and I-b differ only by 3 nucleotides.

the $H$. andersenii mitochondrial genome to see whether a similar pattern exists. Unlike $R$. salina, however, the $H$. andersenii GC skew does not change direction near the repeat region. Instead, in both the $H$. andersenii and $R$. salina mtDNA, observed GC skew patterns strongly correlate with transcriptional orientations, where the coding strand tends to be G-rich (data not shown). Therefore, the GC skew patterns of the two cryptophyte mitochondrial 
Table I: Functional categories of 36 protein genes encoded in the mitochondrial genome of Hemiselmis andersenii.

\begin{tabular}{|c|c|c|}
\hline Protein categories & Sub-categories & Genes \\
\hline \multirow[t]{2}{*}{ Ribosomal proteins (14) } & Small subunit & rps2 rps3 rps 4 rps 7 rps8 rps II rps 12 rps 13 rps 14 rps 19 \\
\hline & Large subunit & rpl5 rpl6 rpll 4 rpll6 \\
\hline \multirow[t]{5}{*}{ Oxidative phosphorylation (2I) } & NADH dehydrogenase & $\begin{array}{l}\text { nadl nad2 nad3 nad4 nad4L nad5 nad6 nad7 nad8 } \\
\text { nad9 nad } 10 \text { nad I I }\end{array}$ \\
\hline & Ubiquinol:cytochrome c oxidoreductase & $c o b$ \\
\hline & Succinate:ubiquinone oxidoreductase & sdh3 \\
\hline & Cytochrome c oxidase & $\operatorname{cox} 1 \operatorname{cox} 2 \operatorname{cox} 3$ \\
\hline & ATP synthase & atp I atp6 atp8 atp9 \\
\hline Sec-independent protein translocase protein (I) & & tatC \\
\hline
\end{tabular}

genomes do not seem to be the result of replication-associated mutational bias, but rather the non-random distribution of the protein coding genes, as has been observed in some other genomes [39]. Nevertheless, based on the presence of other features such as stem-loop structures, it seems reasonable to assume that the repeat region in both cryptophyte mitochondrial genomes corresponds to the origin of replication.

\section{Codon usage and transfer RNAs}

The $H$. andersenii mtDNA encodes 28 tRNAs, 27 of which are predicted to form standard cloverleaf secondary structures. One tRNA gene, 'trnK(uuu)', shows atypical structure in the anticodon loop and the variable region, and is probably a pseudogene (Figure 4A). Allowing for wobble pairings and some base modifications, 26 tRNAs are the theoretical minimum required to cover all codons in bacteria. For some mitochondria, even smaller sets of tRNAs, as few as 22-23, are possible by adopting several additional strategies [40]. The $H$. andersenii mitochondrial genome lacks only one tRNA gene, $\operatorname{trnK}(\mathrm{uuu})$, which is minimally required in order to recognize all 61 codons (Table 3). It is thus predicted that nuclear-encoded cytosolic Lys-tRNA is imported into $H$. andersenii mitochondria. Mitochondrial tRNA import has been demonstrated in apicomplexans and trypanosomatids where tRNA genes are completely missing in their mitochondrial genomes [41], as well as in ciliates and plants where mitochondrial genomes encode fewer than the 22-23 minimally required tRNA genes [42]. Although most animals and some fungi do not import tRNAs into mitochondria
[43], the fungus Saccharomyces cerevisiae has been shown to import one specific cytosolic tRNA even though its mitochondrial genome encodes the full complement of tRNAs [44]. Analyses of the tRNA repertoire of mitochondrial genomes suggest that a number of other protist taxa across the eukaryotic tree also import one or more tRNAs into their mitochondria $[43,45]$. It is thus reasonable to assume that $H$. andersenii imports at least Lys-tRNA, although it is possible that tRNA editing makes up for the Lys-tRNA deficit by changing the identity of an existing tRNA, as has been shown in marsupials [46].

Another possible mechanism to account for the missing tRNA is that the structurally abnormal 'trnK(uuu)' gene (Figure 4A) forms a functional Lys-tRNA to decode the codons AAA and AAG. Several cases of atypically-structured tRNAs are known from animal and ciliate mitochondria [47,48]. Interestingly, tRNAscan-SE [32] predicted the existence of a 20 bp intron within the $H$. andersenii 'trnK(uuu)', and we conducted further experiments to test whether this is indeed the case. RT-PCR experiments using primer sets specific for 'trnK(uuu)' indicated that the putative intron was not removed in the mature tRNA. This results is not unexpected, given that the 20-bp putative intron is too short to be a self-splicing group I or II intron, which are the only known types of introns reported in mitochondrial genomes [49]. Sequencing of $\sim 20$ clones also did not reveal any evidence for RNA editing within the 'trnK(uuu)'. These results suggest that if 'trnK(uuu)' is indeed expressed to form a functional Lys-tRNA, it is predicted to have an unusually AU-

Table 2: Comparison of two cryptophyte mitochondrial genomes

\begin{tabular}{lll}
\hline & Hemiselmis andersenii & Rhodomonas salina \\
\hline Genome size & $60,553 \mathrm{bp}$ & $48,063 \mathrm{bp}$ \\
Coding capacity & $61 \%$ & $69 \%$ \\
GC $\%$ & $29 \%$ & $29 \%$ \\
Size of the repeat region & $19.7 \mathrm{Kbp}(33 \%)$ & $4.7 \mathrm{Kbp}(10 \%)$ \\
Group II introns & not present & two \\
Number of genes (with assignable functions) & 66 genes (28 tRNAs) & 69 genes (27 tRNAs) \\
Inverted repeats & not present & a pair of $\sim 1.5$ Kbp repeat units
\end{tabular}




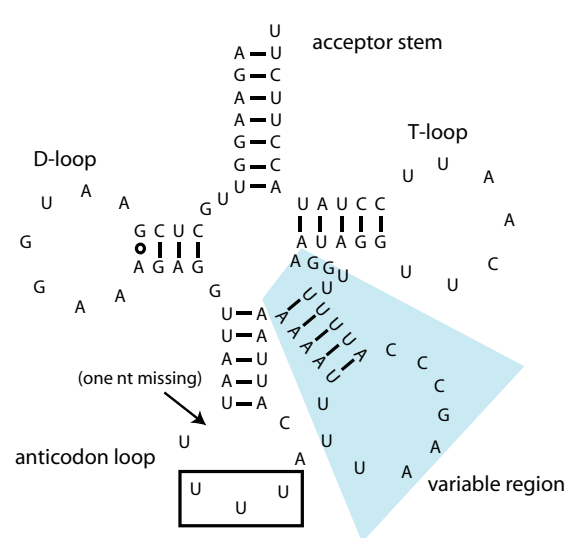

A. H. andersenii tRNA-Lys (uuu) (pseudogene?)

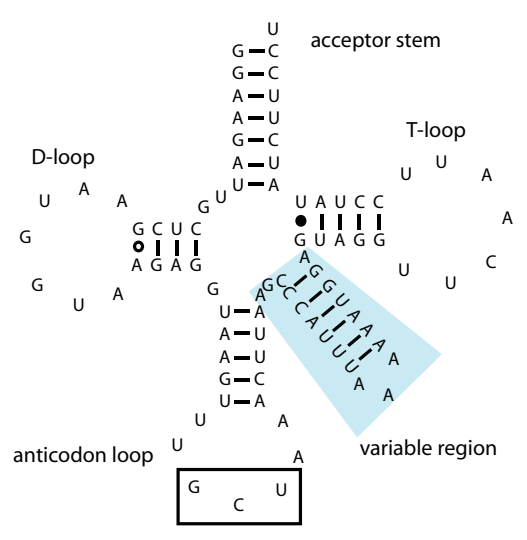

B. H. andersenii tRNA-Ser (gcu)

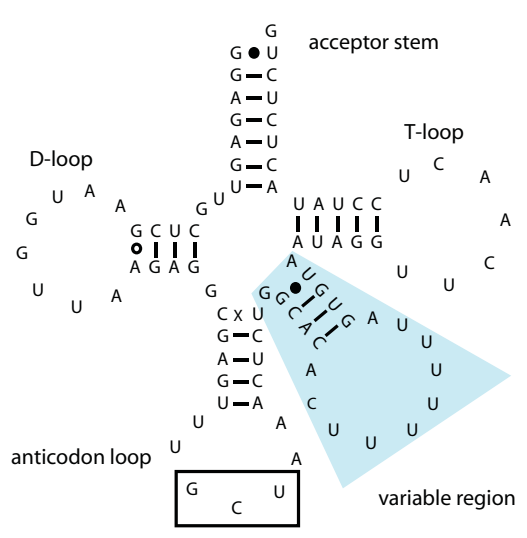

C. R. salina tRNA-Ser (gcu)

\section{Figure 4}

Predicted secondary structures for three homologous cryptophyte tRNAs. Hemiselmis andersenii 'trnK(uuu)' (A) is paralogous to $\operatorname{trnS}(\mathrm{gcu})$ (B). Duplication and divergence of an ancestral trnS(gcu) appears to have led to the evolution of 'trnK(uuu)' in $H$. andersenii (A), which consequently, possesses an atypically long variable region. The anticodon loop and the variable region of 'trnK(uuu)' is AT-rich and the predicted stem regions consist entirely of A-T base pairings. Furthermore, the loop of the anticodon loop/stem region is missing one nucleotide (arrow). These structural considerations suggest that 'trnK(uuu)' may not be functional. The 'trnK(uuu)' gene in H. andersenii (A) is orthologous to trnS(gcu) in R. salina (C). Note that while sequences within the anticodon loop, $\mathrm{V}$-arm, and acceptor stem are divergent between the two orthologous copies, sequences within the D loop and the T loop are conserved.

Table 3: Hemiselmis andersenii mtDNA codon usage table.

\begin{tabular}{|c|c|c|c|c|c|c|c|}
\hline \multirow[b]{3}{*}{ First Position of Codon } & \multicolumn{5}{|c|}{ Second Position of Codon } & & \multirow[b]{3}{*}{ Third Position of Codon } \\
\hline & \multicolumn{2}{|r|}{$U$} & \multirow{2}{*}{$\begin{array}{c}\mathrm{C} \\
\text { UCU }[S] \quad 123\end{array}$} & \multirow{2}{*}{$\frac{A}{U A U[Y] 3 / 2}$} & \multicolumn{2}{|l|}{ G } & \\
\hline & $U$ & UUU [F] 608 & & & UGU $[C] 123$ & $u$ & \\
\hline & & UUC [F] $126{ }^{\circ}$ & UCC $[S] 18$ & UAC [Y] 69॰ & UGC [C] 20• & C & \\
\hline & & UUA [L] 688• & UCA [S] 205• & UAA [stop] 32 & UGA [stop] 2 & $A$ & \\
\hline & & UUG [L] 163 & UCG [S] 57 & UAG [stop] 3 & UGG $[\mathrm{W}] \mathrm{I20}$ & G & \\
\hline & $\mathrm{C}$ & CUU [L] 179 & $\mathrm{CCU}[\mathrm{P}] 139$ & $\mathrm{CAU}[\mathrm{H}] \mathrm{I} 40$ & CGU $[R] 83$ & $u$ & \\
\hline & & CUC [L] 38• & $\mathrm{CCC}[\mathrm{P}] 18$ & $\mathrm{CAC}[\mathrm{H}] 30 \bullet$ & CGC [R] I7• & C & \\
\hline & & CUA [L] $88^{\bullet}$ & $\mathrm{CCA}[\mathrm{P}] 115^{\circ}$ & CAA [Q] $217 \bullet$ & CGA $[R] 95^{\circ}$ & $A$ & \\
\hline & & CUG [L] 20 & CCG $[P] 28$ & CAG [Q] 42 & CGG $[R] 32$ & G & \\
\hline & $A$ & AUU [I] 579 & $A C U[T] 162$ & $\mathrm{AAU}[\mathrm{N}] 307$ & AGU $[S] 183$ & $U$ & \\
\hline & & AUC [I] $85^{\bullet}$ & $A C C[T] 24$ & $\mathrm{AAC}[\mathrm{N}] 95^{\bullet}$ & AGC [S] $29 \bullet^{\circ}$ & C & \\
\hline & & AUA [I] 207• & $\mathrm{ACA}[\mathrm{T}] 242 \bullet$ & AAA $[K] 517 \cdot \dagger$ & $A G A[R] 110$ & $A$ & \\
\hline & & AUG $[M] 233 \cdot \bullet$ & ACG [T] 54 & AAG $[K] 75$ & AGG $[R] 15$ & G & \\
\hline & G & GUU [V] 3II & GCU [A] 186 & GAU [D] 22I & GGU [G] 299 & $U$ & \\
\hline & & GUC [V] 38 & GCC $[A] 35$ & GAC [D] $39^{\circ}$ & GGC [G] $\left.4\right|^{\circ}$ & $\mathrm{C}$ & \\
\hline & & GUA [V] 204• & GCA $[A] 225^{\circ}$ & GAA [E] $283{ }^{\circ}$ & GGA [G] $|3| \bullet$ & $A$ & \\
\hline & & GUG [V] 62 & GCG $[A] 64$ & GAG [E] 65 & GGG [G] 83 & G & \\
\hline
\end{tabular}

The frequency of each codon is shown and dots indicate the presence of a corresponding tRNA gene in the mtDNA. $\dagger:$ 'trnK(uuu)' identified in the genome may be a pseudogene (see discussion). 
rich stem in the codon loop and a long variable region, atypical for Lys-tRNA (Figure 4A). Long variable regions ranging from 11 to 23 nucleotides are generally restricted to tRNA-Leu, tRNA-Ser, and bacterial tRNA-Tyr [40]. The $\mathrm{D}$ - and T-loops of the 'trnK(uuu)' sequence show sequence similarity to one of the two mitochondrion-encoded tRNA-Ser genes (Figure 4A and 4B), both of which have a long variable region. In addition, comparative analysis with the $R$. salina mtDNA revealed genomic position conservation between the $H$. andersenii trnS-like 'trnK(uuu)' gene and the trnS $(\mathrm{gcu})$ gene of $R$. salina, flanked by the tatC and nad7 genes. The $H$. andersenii 'trnK(uuu)' and $R$. salina trnS( $\mathrm{gcu}$ ) genes both overlap tatC by 51 bp and 22 bp, respectively. This strongly suggests that the $H$. andersenii 'trnK(uuu)' is indeed derived from an ancestral gene that encoded tRNA-Ser, explaining the origin of its long variable region. The overlap between the $H$. andersenii 'trnK(uuu)' and tatC suggests that 'trnK(uuu)' may play a role in processing the 3 ' end of the tat $C$ gene transcript. This hypothesis could explain why the 'trnK(uuu)' gene still remains in the genome and retains conserved secondary structure in the stem loop and D- and T-loops, even if it does not form a functional tRNA. Comprehensive molecular and biochemical experimentation will be necessary to confirm or refute the existence of mitochondrial tRNA import in $H$. andersenii and the functionality of the unusual 'trnK(uuu)' gene.

When the $H$. andersenii tRNA genes were compared to those of $R$. salina, 24 homologous pairs of tRNAs were identified, leaving only four $H$. andersenii tRNA and three $R$. salina tRNA genes not unambiguously matched to each other. Each of the tRNA pairs possess identical anticodons except for the $H$. andersenii 'trnK(uuu)' and $R$. salina $\operatorname{trnS}(\mathrm{gcu})$ pair, despite their common derivation. The $\operatorname{trnS}(\mathrm{gcu})$ of $H$. andersenii, having sequence homology to the 'trnK(uuu)', probably originated from a recent gene duplication event. Of the three remaining $H$. andersenii tRNA genes that are unmatched in R. salina, two-trnL (gag) and $\operatorname{trn} G(g c c)$-are redundant because trnL(uag) and $\operatorname{trn} G(u c c)$ can decode all of their respective four-codon families [40]. These redundant copies might have been lost in an ancestor of $R$. salina after it diverged from $H$. andersenii. Lastly, the $H$. andersenii trnI(cau) is somewhat similar to the $\operatorname{trnK}(\mathrm{uuu})$ of the R. salina and only marginally resembles the R. salina trnI(cau) at the 3 ' end. It is possible that the $H$. andersenii $\operatorname{trnI}(\mathrm{cau})$ originated through recombination between ancestral trnI(cau) and trnK(uuu) genes, which would explain the lack of an obvious trnK(uuu) homolog in $H$. andersenii comparable to the $R$. salina trnK(uuu). Substantial sequence divergence among the three genes, however, makes it difficult to accurately trace the origin of the $\operatorname{trnI}(\mathrm{cau})$ and the loss of the original $\operatorname{trnK}(\mathrm{uuu})$ gene in $H$. andersenii. On the other hand, the unusual trnI(uau) gene reported from $R$. salina is not found in $H$. andersenii. It was suggested that the $R$. salina trnI(uau) is derived from $\operatorname{trnF}(\mathrm{uuc})$ through a recent gene duplication event [24]. Overall, the two cryptophyte mitochondrial genomes use similar tRNA sets to recognize codons. However, unlike $H$. andersenii, which may need to import at least $\operatorname{trnK(uuu)}$ from cytosol, the $R$. salina mtDNA does possess the minimal required set for tRNA autonomy.

\section{Molecular phylogenetic analyses}

Cryptophytes are a well-established eukaryotic lineage, supported by both molecular and morphological features [20]. However, their relationship to other eukaryotic groups, particularly those containing plastids of secondary endosymbiotic origin, has been the subject of considerable debate. The cryptophyte plastid is the product of a secondary endosymbiosis involving a red algal cell, the same process which accounts for plastid origins in haptophytes, dinoflagellates, and stramenopiles [50]. CavalierSmith [50] suggested that plastids in these four algal lineages arose from a single secondary endosymbiosis in a common ancestor that these organisms shared, to the exclusion of other eukaryotic groups. However, this "chromalveolate" hypothesis is controversial [51,52]. Recent molecular studies have shown that the katablepharids, an enigmatic collection of plastid-less flagellates, are a sister group to cryptophytes $[53,54]$, and large-scale concatenated analyses of nuclear genes suggest that cryptophytes and haptophytes are also related $[55,56]$.

To gain insight into the phylogenetic relationship of the cryptophytes $H$. andersenii and $R$. salina to other eukaryotes, and more specifically, to test the hypothesis that cryptophytes and haptophytes are related to one another, phylogenetic analyses of mitochondrial protein sequences were performed (Figure 5). Unlike the cryptophyte plastid genome, in which several cases of LGT have recently been discovered $[57,58]$, individual analyses of 25 mitochondrial proteins did not reveal any obvious instances of LGT between prokaryotes and eukaryotes or within eukaryotes (data not shown). However, the possibility of ancient LGTs cannot be ruled out, as the backbones of individual protein phylogenies were generally very poorly supported.

As expected, a close relationship between the two cryptophytes $H$. andersenii and $R$. salina was well supported in the mitochondrial protein phylogenies, with twenty of twenty-five individual protein phylogenies showing this relationship. Five individual gene phylogenies-nad2, $r p l 14, r p l 16, r p s 12, r p s 14-$ did not recover a $H$. andersenii- $R$. salina clade, although alternative topologies were not supported with $>50 \%$ bootstrap support values. Additionally, single protein phylogenies were not, for the most part, able to resolve the relationship of cryptophytes to other eukaryotes. The position of cryptophytes was highly vari- 


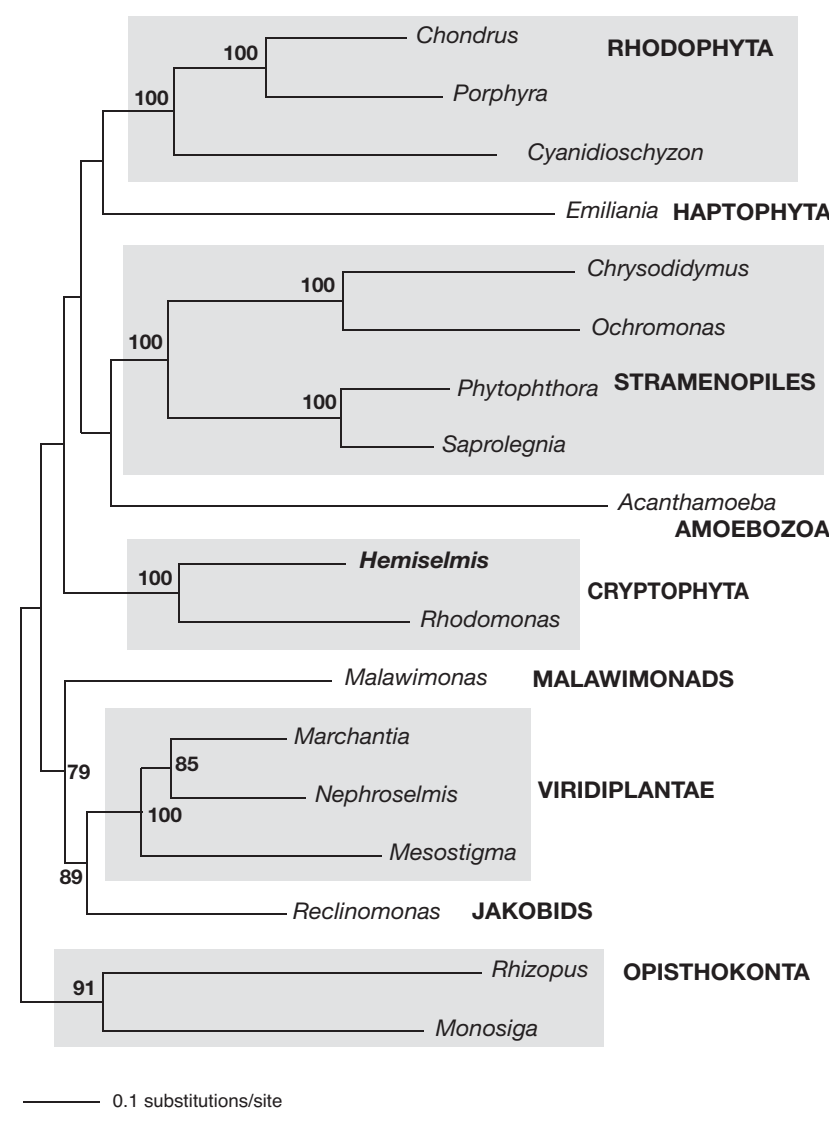

Figure 5

Maximum likelihood phylogenetic tree based on 25 concatenated mitochondrial protein sequences inferred using RAxML. Approximately 15\% of data are missing. Bootstrap support values over $50 \%$ are indicated at the corresponding nodes.

able from protein to protein and the group did not regularly associate with other taxonomic clades with $>50 \%$ bootstrap support values, except for in the cob and nad1 gene trees, where cryptophytes branch with haptophytes $(81 \%)$ and jakobids (77\%), respectively.

We subsequently analyzed a set of 25 concatenated proteins to assess the phylogenetic position of cryptophytes. In this analysis, the $H$. andersenii- $R$. salina clade received $100 \%$ bootstrap support (Figure 5). Other well-established eukaryotic groups including opisthokonts, rhodophytes, stramenopiles, and Viridiplantae, were also strongly recovered, but the relationships among major lineages were not. The jakobid Reclinomonas branched as the sister group to the Viridiplantae with moderate support (89\% bootstrap support), and Malawimonas showed an affinity for these two groups in two of the three data sets, as was previously inferred from a concatenate of ten mitochondrial proteins [59]. It is not clear whether the jabokid (and/or malawimonad)-Viridiplantae affinity is a phylogenetic artifact or reflects the true evolutionary history of mitochondrial genes. Though growing evidence supports a relationship between cryptophytes and haptophytes $[55,56,58]$, our extensive mitochondrial protein analyses did not reveal this relationship with reasonable bootstrap support, other than in a single protein gene tree $(c o b)$. In summary, while mitochondrial gene sequences are able to resolve some of the eukaryotic lineages determined using other markers, they are at present incapable of resolve the deepest branches of the eukaryotic tree using current phylogenetic methods and with the present level of taxon sampling.

\section{Conclusion}

We have sequenced the mitochondrial genome of the cryptophyte $H$. andersenii and compared it to that of the distantly related cryptophyte $R$. salina. Our analyses reveal that both genomes are characterized by a gene dense region and a single large intergenic space that includes numerous repeats and palindromic sequences predicted to form stable DNA stem and loop structures. Despite the overall similarities in content and architecture between the two genomes, their modes of regulating DNA replication and transcription seem to differ. Unlike R. salina, all 73 genes in the $H$. andersenii mtDNA are located on the same strand, a relatively rare observation in mitochondrial genomes. Phylogenic analysis of multiple mitochondrial gene sequences indicated a clear affiliation between the two cryptophytes but was not able to resolve the position of cryptophytes relative to other eukaryotic groups.

\section{Authors' contributions}

EK participated in genome assembly, carried out genome analysis and drafted the manuscript. CEL isolated $H$. andersenii DNA and participated in the initial genome assembly. BAC, $\mathrm{CK}$, and SB performed the $H$. andersenii mitochondrial genome sequencing. JMA coordinated the study and helped draft the manuscript. All authors read and approved the manuscript.

\section{Acknowledgements}

We thank D. Spencer for discussion, J. Leigh for mitochondrial protein sequence alignments, $H$. Khan for $H$. andersenii RNA, A. Roger for help with phylogenetic analyses, and D. Spencer and $H$. Khan for helpful comments on the manuscript. A. Bendich is acknowledged for providing insight on the probable in vivo structure of $H$. andersenii mtDNA. This work was supported by Genome Atlantic and a Natural Sciences and Engineering Research Council of Canada Discovery Grant (28335-04) awarded to JMA. EK receives postdoctoral fellowship support from the Tula Foundation. JMA is a Scholar of the Canadian Institute for Advanced Research, Program in Integrated Microbial Biodiversity.

\section{References}

I. Burger G, Gray MW, Lang BF: Mitochondrial genomes: anything goes. Trends Genet 2003, I 9(12):709-716. 
2. Tovar J, Leon-Avila G, Sanchez LB, Sutak R, Tachezy J, van der Giezen $M$, Hernandez M, Muller M, Lucocq JM: Mitochondrial remnant organelles of Giardia function in iron-sulphur protein maturation. Nature 2003, 426(6963): I72-I76.

3. Marande W, Burger G: Mitochondrial DNA as a genomic jigsaw puzzle. Science 2007, 3 I 8(5849):4I5-4I5

4. Slamovits $\mathrm{CH}$, Saidarriaga JF, Larocque A, Keeling PJ: The highly reduced and fragmented mitochondrial genome of the earlybranching dinoflagellate Oxyrrhis marina shares characteristics with both apicomplexan and dinoflagellate mitochondrial genomes. J Mol Biol 2007, 372(2):356-368.

5. Nosek J, Tomaska L: Mitochondrial genome diversity: evolution of the molecular architecture and replication strategy. Curr Genet 2003, 44(2):73-84.

6. Burger G, Zhu Y, Littlejohn TG, Greenwood SJ, Schnare MN, Lang BF, Gray MW: Complete sequence of the mitochondrial genome of Tetrahymena pyriformis and comparison with Paramecium aurelia mitochondrial DNA. I Mol Biol 2000, 297(2):365-380

7. Vahrenholz C, Riemen G, Pratje E, Dujon B, Michaelis G: Mitochondrial DNA of Chlamydomonas reinhardtii: the structure of the ends of the linear $15.8 \mathrm{~Kb}$ genome suggests mechanisms for DNA replication. Curr Genet 1993, 24(3):24I-247.

8. Shao ZY, Graf S, Chaga OY, Lavrov DV: Mitochondrial genome of the moon jelly Aurelia aurita (Cnidaria, Scyphozoa): A linear DNA molecule encoding a putative DNA-dependent DNA polymerase. Gene 2006, 381:92-101.

9. Bendich AJ: Reaching for the ring: the study of mitochondrial genome structure. Curr Genet 1993, 24(4):279-290.

10. Bendich AJ: Structural analysis of mitochondrial DNA molecules from fungi and plants using moving pictures and pulsed-field gel electrophoresis. J Mol Biol 1996, 255(4):564-588.

II. Marande W, Lukes J, Burger G: Unique mitochondrial genome structure in diplonemids, the sister group of kinetoplastids. Eukaryot Cell 2005, 4(I 2):2I 70-2 I 70.

12. Lang BF, Burger G, O'Kelly CJ, Cedergren R, Golding GB, Lemieux C, Sankoff D, Turmel M, Gray MW: An ancestral mitochondria DNA resembling a eubacterial genome in miniature. Nature 1997, 387(6632):493-497.

13. Ji YE, Mericle BL, Rehkopf DH, Anderson JD, Feagin JE: The Plasmodium falciparum 6 kb element is polycistronically transcribed. Mol Biochem Parasitol 1996, 8 I (2):2 II-223.

14. Clemens DL, Johnson PJ: Failure to detect DNA in hydrogenosomes of Trichomonas vaginalis by nick translation and immunomicroscopy. Mol Biochem Parasitol 2000, 106(2):307-3I3.

15. Embley TM, Martin W: Eukaryotic evolution, changes and challenges. Nature 2006, 440(7084):623-630.

16. Maslov DA, Avila HA, Lake JA, Simpson L: Evolution of RNA editing in kinetoplastid protozoa. Nature 1994, 368(6469):345-348.

17. Knoop V: The mitochondrial DNA of land plants: peculiarities in phylogenetic perspective. Curr Genet 2004, 46(3): 123-I39.

18. Zhang $\mathrm{H}$, Lin S: Mitochondrial cytochrome $b$ mRNA editing in dinoflagellates: possible ecological and evolutionary associations? J Eukaryot Microbiol 2005, 52(6):538-545

19. Lin SJ, Zhang HA, Spencer DF, Norman JE, Gray MW: Widespread and extensive editing of mitochondrial mRNAs in dinoflagellates. J Mol Biol 2002, 320(4):727-739.

20. Graham LE, Wilcox LW: Algae. Upper Saddle River, NJ, Prentice Hall; 2000.

21. Adl SM, Simpson AGB, Farmer MA, Andersen RA, Anderson OR, Barta JR, Bowser SS, Brugerolle G, Fensome RA, Fredericq S, James TY, Karpov S, Kugrens P, Krug J, Lane CE, Lewis LA, Lodge J, Lynn DH, Mann DG, McCourt RM, Mendoza L, Moestrup O, Mozley-Standridge SE, Nerad TA, Shearer CA, Smirnov AV, Spiegel FW, Taylor MFJR: The new higher level classification of eukaryotes with emphasis on the taxonomy of protists. J Eukaryot Microbiol 2005 52(5):399-45I.

22. Archibald JM: Nucleomorph genomes: structure, function, origin and evolution. Bioessays 2007, 29(4):392-402.

23. Douglas S, Zauner S, Fraunholz M, Beaton M, Penny S, Deng LT, Wu $X N$, Reith M, Cavalier-Smith T, Maier UG: The highly reduced genome of an enslaved algal nucleus. Nature 200I, 410(6832): $109 \mid-1096$.

24. Hauth AM, Maier UG, Lang BF, Burger G: The Rhodomonas salina mitochondrial genome: bacteria-like operons, compact gene arrangement and complex repeat region. Nucleic Acids Res 2005, 33( I 4):4433-4442.

25. Lane CE, van den Heuvel K, Korera C, Curtis BA, Parsons B], Bowman S, Archibald JM: Nucleomorph genome of Hemiselmis andersenii reveals complete intron loss and compaction as a driver of protein structure and function. Proc Natl Acad Sci U S A 2007, 104:19908-19913.

26. Staden R, Beal KF, Bonfield JK: The Staden package, 1998. Methods Mol Biol 2000, I32:1 I5-I30.

27. Maddison DR, Maddison WP: MacClade 4: analysis of phylogeny and character evolution. Sunderland, MA, Sinauer Associates Inc.; 2001 .

28. Zuker M: Mfold web server for nucleic acid folding and hybridization prediction. Nucleic Acids Res 2003, 3 I (I 3):3406-34 I 5.

29. Lane CE, Khan H, MacKinnon M, Fong A, Theophilou S, Archibald JM: Insight into the diversity and evolution of the cryptomonad nucleomorph genome. Mol Biol Evol 2006, 23(9):1817-1817.

30. Lane CE, Archibald JM: Novel nucleomorph genome architecture in the cryptomonad genus Hemiselmis. J Eukaryot Microbiol 2006, 53(6):5|5-52|.

3I. Rutherford K, Parkhill J, Crook J, Horsnell T, Rice P, Rajandream MA, Barrell B: Artemis: sequence visualization and annotation. Bioinformatics 2000, I6( I 0):944-945.

32. Lowe TM, Eddy SR: tRNAscan-SE: a program for improved detection of transfer RNA genes in genomic sequence. Nucleic Acids Res 1997, 25(5):955-964.

33. Tesler G: GRIMM: genome rearrangements web server. Bioinformatics 2002, I 8(3):492-493.

34. Stamatakis A: RAxML-VI-HPC: maximum likelihood-based phylogenetic analyses with thousands of taxa and mixed models. Bioinformatics 2006, 22(2I):2688-2690.

35. Burger G, Plante I, Lonergan KM, Gray MW: The mitochondria DNA of the ameboid protozoan, Acanthamoeba castellanii: complete sequence, gene content and genome organization. J Mol Biol 1995, 245(5):522-537.

36. Woo PCY, Zhen HJ, Cai JJ, Yu J, Lau SKP, Wang J, Teng JLL, Wong SSY, Tse RH, Chen R, Yang HM, Liu B, Yuen KY: The mitochondrial genome of the thermal dimorphic fungus Penicillium marneffei is more closely related to those of molds than yeasts. FEBS Lett 2003, 555(3):469-477.

37. Denovanwright EM, Lee RW: Comparative structure and genomic organization of the discontinuous mitochondrial ribosomal RNA genes of Chlamydomonas eugametos and Chlamydomonas reinhardtii. J Mol Biol 1994, 24 I(2):298-3II.

38. Turmel M, Lemieux C, Burger G, Lang BF, Otis C, Plante I, Gray MW: The complete mitochondrial DNA sequences of Nephroselmis olivacea and Pedinomonas minor. Two radically different evolutionary patterns within green algae. Plant Cell 1999, II(9): 1717-1730.

39. Mclean M], Wolfe KH, Devine KM: Base composition skews, replication orientation, and gene orientation in 12 prokaryote genomes. J Mol Evol 1998, 47(6):69|-696.

40. Marck C, Grosjean H: tRNomics: analysis of tRNA genes from 50 genomes of Eukarya, Archaea, and Bacteria reveals anticodon-sparing strategies and domain-specific features. RNA 2002, 8(i 0): $1189-1232$.

41. Esseiva AC, Naguleswaran A, Hemphill A, Schneider A: Mitochondrial tRNA import in Toxoplasma gondii. J Biol Chem 2004, 279(4I):42363-42368.

42. Glover KE, Spencer DF, Gray MW: Identification and structural characterization of nucleus-encoded transfer RNAs imported into wheat mitochondria. J Biol Chem 200I, 276(I):639-648.

43. Schneider A, Marechal-Drouard L: Mitochondrial tRNA import: are there distinct mechanisms? Trends Cell Biol 2000, I0(I 2):509-513.

44. Hopper AK, Phizicky EM: tRNA transfers to the limelight. Genes Dev 2003, 17(2): 162-180.

45. Gray MW, Lang BF, Cedergren R, Golding GB, Lemieux C, Sankoff D, Turmel M, Brossard N, Delage E, Littlejohn TG, Plante I, Rioux P, Saint-Louis D, Zhu Y, Burger G: Genome structure and gene content in protist mitochondrial DNAs. Nucleic Acids Res 1998, 26(4):865-878.

46. Borner GV, Morl M, Janke A, Paabo S: RNA editing changes the identity of a mitochondrial tRNA in marsupials. $E M B O$ ] 1996, I5(2I):5949-5957. 
47. Schnare MN, Greenwood SJ, Gray MW: Primary sequence and posttranscriptional modification pattern of an unusual mitochondrial tRNA(Met) from Tetrahymena pyriformis. FEBS Lett 1995, 362(I):24-28.

48. Steinberg $S$, Cedergren R: Structural compensation in atypical mitochondrial transfer RNAs. Nat Struct Biol I994, I (8):507-510.

49. Lang BF, Laforest MJ, Burger G: Mitochondrial introns: a critical view. Trends Genet 2007, 23(3): 1 19-125.

50. Cavalier-Smith T: Principles of protein and lipid targeting in secondary symbiogenesis: euglenoid, dinoflagellate, and sporozoan plastid origins and the eukaryote family tree. J Eukaryot Microbiol 1999, 46(4):347-366.

5I. Grzebyk D, Katz ME, Knoll AH, Quigg A, Raven JA, Schofield O, Taylor FJR, Falkowski PG: Response to comment on "The evolution of modern eukaryotic phytoplankton". Science 2004, 306(5705):219lc.

52. Keeling PJ, Archibald JM, Fast NM, Palmer JD: Comment on "The evolution of modern eukaryotic phytoplankton". Science 2004, 306(5705):219lb.

53. Kim E, Simpson AGB, Graham LE: Evolutionary relationships of apusomonads inferred from taxon-rich analyses of 6 nuclear encoded genes. Mol Biol Evol 2006, 23(I 2):2455-2466.

54. Okamoto N, Inouye I: The katablepharids are a distant sister group of the Cryptophyta: a proposal for Katablepharidophyta divisio nova/Kathablepharida phylum novum based on SSU rDNA and beta-tubulin phylogeny. Protist 2005, 156(2):163-179.

55. Hackett JD, Yoon HS, Li S, Reyes-Prieto A, Rummele SE, Bhattacharya D: Phylogenomic analysis supports the monophyly of cryptophytes and haptophytes and the association of Rhizaria with Chromalveolates. Mol Biol Evol 2007, 24(8): I702-1713.

56. Patron NJ, Inagaki Y, Keeling PJ: Multiple gene phylogenies support the monophyly of cryptomonad and haptophyte host lineages. Curr Biol 2007, I7(1 0):887-89I.

57. Khan H, Parks N, Kozera C, Curtis BA, Parsons BJ, Bowman S, Archibald JM: Plastid genome sequence of the cryptophyte alga Rhodomonas salina CCMP I 3 | 9: lateral transfer of putative DNA replication machinery and a test of chromist plastid phylogeny. Mol Biol Evol 2007, 24(8): I832-1842.

58. Rice DW, Palmer JD: An exceptional horizontal gene transfer in plastids: gene replacement by a distant bacterial paralog and evidence that haptophyte and cryptophyte plastids are sisters. BMC Biol 2006, 4:3 I.

59. Secq MPO, Goer SL, Stam WT, Olsen JL: Complete mitochondrial genomes of the three brown algae (Heterokonta : Phaeophyceae) Dictyota dichotoma, Fucus vesiculosus and Desmarestia viridis. Curr Genet 2006, 49(1):47-58. 